\title{
Expression analysis of the TAB2 protein in adult mouse tissues
}

\author{
C. Orelio and E. Dzierzak \\ Erasmus University Medical Centre, Dept. of Cell Biology and Genetics, P.O. Box 1738, 3000 DR Rotterdam, The Netherlands, \\ Fax: ++31 10408 9468, e-mail: e.dzierzak@erasmusmc.nl \\ Received 10 April 2006; returned for revision 1 September 2006; accepted by J. Di Battista 13 September 2006
}

\begin{abstract}
Background: The Interleukin-1 (IL-1) signaling component TAK1 binding protein 2 (TAB2) plays a role in activating the NFKB and JNK signaling pathways. Additionally, TAB2 functions in the nucleus as a repressor of NFkBmediated gene regulation.

Objective: To obtain insight into the function of TAB2 in the adult mouse, we analyzed the in vivo TAB2 expression pattern.

Materials and methods: Cell lines and adult mouse tissues were analyzed for TAB2 protein expression and localization.

Results: Immunohistochemical staining for TAB2 protein revealed expression in the vascular endothelium of most tissues, hematopoietic cells and brain cells. While TAB2 is localized in both the nucleus and the cytoplasm in cell lines, cytoplasmic localization predominates in hematopoietic tissues in vivo.

Conclusions: The TAB2 expression pattern shows striking similarities with previously reported IL-1 receptor expression and NFkB activation patterns, suggesting that TAB2 in vivo is playing a role in these signaling pathways.
\end{abstract}

Key words:TAB2-Map3k7ip2-NF-kappaB-Interleukin-1Hematopoiesis

\section{Introduction}

The cytokine Interleukin-1 (IL-1) is a well-known regulator within the hematopoietic system. IL-1 can act on several different hematopoietic cell types and plays a role in regulating their proliferation and differentiation, depending on the target cell and the presence of additional cytokines [1-3]. IL-1 has also been shown to play a role in the migration and radioprotection of immature hematopoietic cells $[4,5]$, indicating

Support was provided by the ZonMW VICI (916.36.601), NIH (RO1 DK51077)

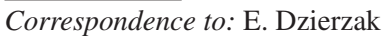

its versatile role within the hematopoietic system. Moreover, IL-1 plays an important role in inflammatory reactions [6]. $\mathrm{IL}-1$ is a potent inducer of fever and acute phase response in the adult [3] and importantly, IL-1 has been implicated in several inflammatory-related diseases, such rheumatoid arthritis, the neurodegenerative Alzheimer's disease and in vascular diseases such as atherosclerosis [7-9].

The regulation of these different biological processes by IL-1 is mediated via signalling from the IL-1RI towards downstream signalling pathways, including the NFKB pathway [6]. Signalling via NFkB members results in the regulated expression of various genes including those of cytokines and receptors involved in hematopoiesis and inflammation, as well as apoptosis and cell adhesion $[10,11]$. NFkB signalling induced by IL-1 in concert with other signals from the IL-1R and other cytokine receptors determines the biological outcome of the IL-1 induced response.

Activation of the NFkB pathway by IL- 1 is mediated via the transforming growth factor $\beta$ (TGF- $\beta$ ) activated kinase 1 (TAK1; also known as mitogen activated protein 3 kinase 7 (MAP3K7)) together with three TAK1 binding proteins (TAB1, TAB2 and TAB3). TAB1 is a regulator of TAK1 kinase activity and is widely expressed $[12,13]$. TAB2 was originally reported as an adapter protein required for the recruitment of TAK1 and TRAF6 to the IL-1 receptor. More recent studies showed that TAB2 plays a similar role in the LPS, Toll and TNF signaling pathways [14-16]. TAB3 is highly homologous to TAB2 and also functions as an adapter protein linking TAK1 to similar receptors as reported for TAB2 $[15,17,18]$.

The role for TAB2 as an adapter protein has been questioned by more recent studies with TAB 2 deficient cells and mice $[19,20]$. Upon IL-1 stimulation, TAB2-deficient cells responded similarly to wildtype cells in the expression of IL1 target genes and the activation of the cytoplasmic JNK and NFkB signaling pathways.

Besides its role as a TAK1 adapter protein, another role for TAB2 was provided by studies in which TAB2 was shown to act as modulator of NFkB-mediated gene activation in the nucleus $[21,22]$. Upon IL-1 stimulation, TAB2 was transported from the nucleus to the cytoplasm and TAB2-mediated repression of $\mathrm{NFkB}$ target genes was 
thereby relieved. Altogether, these different studies show that TAB2 could be playing different roles in IL-1 receptor signaling and NFkB-mediated gene regulation depending on the subcellular localization of TAB2.

Although the cellular pattern associated with IL-1 receptor expression and NFkB activation in the adult mouse tissues [23] is known, little information is available concerning the expression pattern of TAB2 or the role it plays in physiological processes. As the expression pattern of TAB2 could provide more insight into its in vivo role in IL-1 and NFkB signaling, we studied the TAB2 expression in the adult mouse. Protein analysis revealed TAB2 expression in all adult tissues examined, with a distinctive spatial expression of TAB2 in brain, thymus and spleen.

\section{Material and methods}

\section{Mice, cell enrichment and cell culture}

Animals were housed according to institutional guidelines, with free access to water and food. Animal procedures were carried out in compliance with the Standards for Humane Care and Use of Laboratory Animals. (CBAxC57/BL10)F1 mice used for these studies were sacrificed by cervical dislocation and tissues collected in PBS/10\%FCS/1\% Pen/Strep.

Bone marrow and spleen cells were incubated with PE-conjugated anti-CD45 antibody (Pharmingen) for $20 \mathrm{~min}$ on ice, washed twice and resuspended in PBS/10\% FCS/1 \% Pen/Strep and Hoechst $33258(1 \mu \mathrm{g} /$ $\mathrm{ml}$, Molecular Probes) for dead cell exclusion. Cells were sorted on a FACSAria (Becton Dickinson). To separate mature (lineage positive) and immature (lineage negative) BM fractions, hematopoietic cells were incubated with CD11b, CD5, Gr-1, Ter119, B220 (Pharmingen en Immunotech) followed by incubation with magnetic microbeads (Miltenyi Biotec). Lineage positive and negative cells were sorted on the autoMACS system (Miltenyi Biotec).

3 T3 fibroblasts were cultured in DMEM/10\% FCS/1 \% PenStrep and CHO cells were cultured with Ham's F10/10\%FCS/1 \% PenStrep. Cells were transfected with either Lipofectamine $2000^{\mathrm{TM}}$ (Life Technologies) or Fugene Transfection reagent ${ }^{\mathrm{TM}}$ (Roche) according to manufacturer's instructions and harvested 24-48 h after transfection.

\section{Generation of the HA-tagged mTAB2 constructs}

The following primers were used to amplify the mouse TAB2 cDNA from the TAB2 IMAGE clone (3586132) and to introduce a BglII restriction site upstream of the ATG-start codon: TAB2 forw $5^{\prime}$-AGATCTATGGCCCAAGGAAGCCACC-3' and TAB2 rev 5'AGAAATGCCGAGGCATCTCAC-3'. The mTAB2 cDNA was cloned into PGEM-Teasy vector ${ }^{\mathrm{TM}}$ (Promega) and confirmed by sequencing. mTAB2 was subcloned into the pBluescript vector ${ }^{\mathrm{TM}}$ (Stratagene) and a HA-tag (a kind gift of D.Meier) was inserted in frame with the coding region of mTAB2 cDNA. Thereafter HA-mTAB2 was cloned into the pCDNA ${ }^{\mathrm{TM}}$ expression vector (Invitrogen) for overexpression studies. The N-terminally truncated mTAB2 construct was initially PCR-amplified from the mTAB2 IMAGE clone with the following primers TAB21280 forw 5'-AGATCTATGCGGAATCAACCGACACTCTTC-3' and TAB2 rev 5'AGAAATGCCGAGGCATCTCAC-3' and the construct was generated in a similar manner as described above.

\section{Western blotting}

Protein lysates were made with RIPA lysis buffer or in Laemlli sample buffer. Protein lysates derived from an equivalent of $0.4-2 \times 10^{6}$ cells per tissue were separated on 7-10\% SDS-PAGE and blotted to PVDF membrane (Immobilon, Millipore). Blotted membranes were blocked with $4 \%$ non-fat dry milk (Biorad)/ TBS-T and incubated overnight with primary antibody in $2 \%$ milk/TBS-T, then incubated with HRP labeled secondary antibody and visualized by ECL detection. Antibodies used were TAB2 (against C-terminus, k-20; Tebu Bio), TAB2 (against C-terminus, PA1-853 Affinity BioReagents), $\alpha$-HA (Santa Cruz), and anti-actin (Sigma).

\section{Immunofluorescence and histochemistry}

Adult tissues were snap-frozen and $7-10 \mu \mathrm{M}$ cryosections were made. Sections were fixed in $2 \%$ paraformaldehyde/PBS and incubated with primary antibody overnight at $4{ }^{\circ} \mathrm{C}$. Staining was visualized with Santa Cruz or Vector Laboratories ABC staining kit in combination with TSA biotin system (PerkinElmer) according to provided instructions. Sections were counterstained with hematoxylin, dehydrated and embedded in Entallan.

For immunostaining studies, cytospins of BM and spleen cells were made or cells were grown on gelatin-coated glass coverslips. Cells were fixed with $4 \%$ paraformaldehyde and stained with above described antibodies diluted in NETGEL (50 mM Tris pH 7.4, $150 \mathrm{mM} \mathrm{NaCl}, 5 \mathrm{mM}$ EDTA, $0.05 \%(\mathrm{v} / \mathrm{v}) \mathrm{NP}-40,0.25 \%(\mathrm{w} / \mathrm{v})$ gelatin, $0.02 \%(\mathrm{w} / \mathrm{v}) \mathrm{Na}-$ azide). Staining was visualized with secondary FITC or Oregon Greenlabelled antibodies (Molecular Probes) and nuclei were counterstained with DAPI or propidiumiodide.

\section{Results}

Cytoplasmic and nuclear localization of overexpressed $H A-T A B 2$

In our initial studies examining mTAB2 expression, we observed only low levels of protein in most cells and tissues. To confirm that we were indeed detecting TAB2, to verify its molecular weight and to determine its subcellular localization, we generated HA-tagged mTAB2 constructs that could be used for overexpression studies. Transient overexpression of HA-tagged full length and N-terminally truncated mTAB2 constructs (Fig. 1A) in 3T3 fibroblast or $\mathrm{CHO}$ cells was followed by Western blotting to detect the HA-tagged proteins. As a positive control for the HA antibody, we used a lysate from cells expressing HA-tagged Oct6 protein. As shown in Figure 1B, the protein lysate from HA-mTAB2 full length transfected cells yielded a band on Western blot with a molecular weight of approximately $80 \mathrm{kD}$, consistent with the expected size of the mTAB2 protein with the HA-tag ( $76 \mathrm{kD}$ plus $3 \mathrm{kD})$. Additionally, overexpression of the truncated HA-mTAB2C construct yielded the expected smaller protein product (approximately $37 \mathrm{kD}$ ) on Western blot.

Since the subcellular localization of a protein can provide an insight into its cellular function, we examined the localization of HA-tagged mTAB2 in $\mathrm{CHO}$ cells. Previously, the N-terminal truncated mTAB2C protein was reported to act in a dominant negative manner in the IL-1 and TNF signaling pathways [16]. Thus, we examined whether the N-terminal truncated and full-length mTAB2 proteins were localized to similar cellular compartments. As shown in Figure $1 \mathrm{C}$, we found a punctate staining pattern in the cytoplasm of mTAB2 overexpressing $\mathrm{CHO}$ cells. In some overexpressing cells, we also observed nuclear mTAB2 


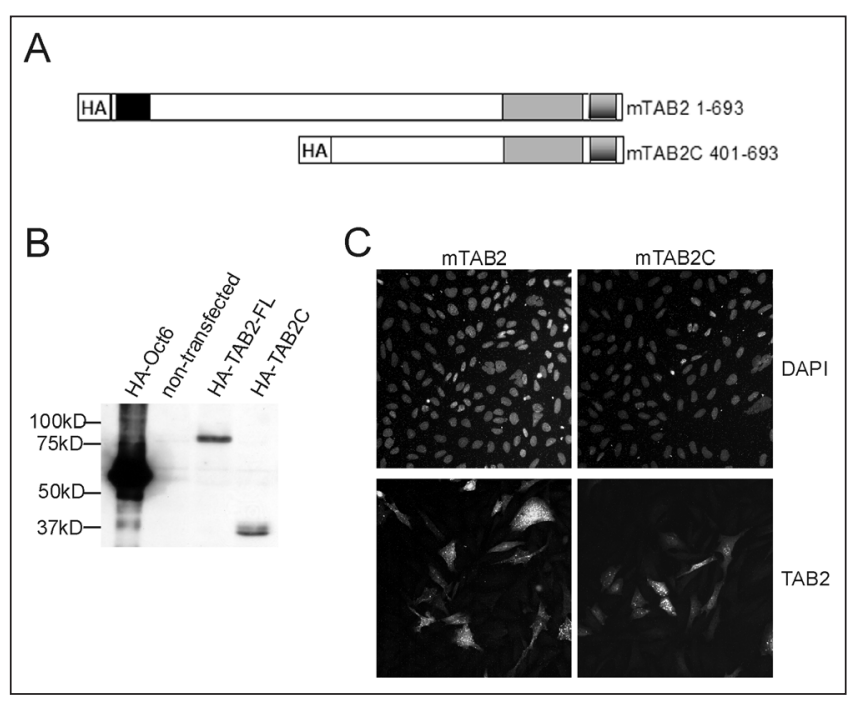

Fig. 1. HA-mTAB2 overexpression in cell lines. A: Schematic overview of the HA-mTAB2 constructs generated and used for overexpression studies in cell lines. The protein domains of TAB2 are indicated and include an N-terminal CUE domain, a coiled-coil domain and an N-terminal Zinc Finger $(\mathrm{ZnF})$ domain. The TAB2C truncated protein has been shown to act as a dominant negative TAB2 mutant protein. B: Western blot probed with anti-HA specific antibody shows overexpression of HA-TAB2 full length (FL) and truncated protein (TAB2C) in 3T3 fibroblasts. These TAB 2 constructs express a $78 \mathrm{kD}$ and $37 \mathrm{kD}$ protein respectively. C: Immunofluorescence of transfected $\mathrm{CHO}$ cells overexpressing HA-tagged mTAB2 full-length and truncated (mTAB2C) protein. DAPI and anti-HA antibody stainings show that mTAB2 protein is localized in a punctate pattern in the cytoplasm and nucleus. Original magnification $400 \times$.

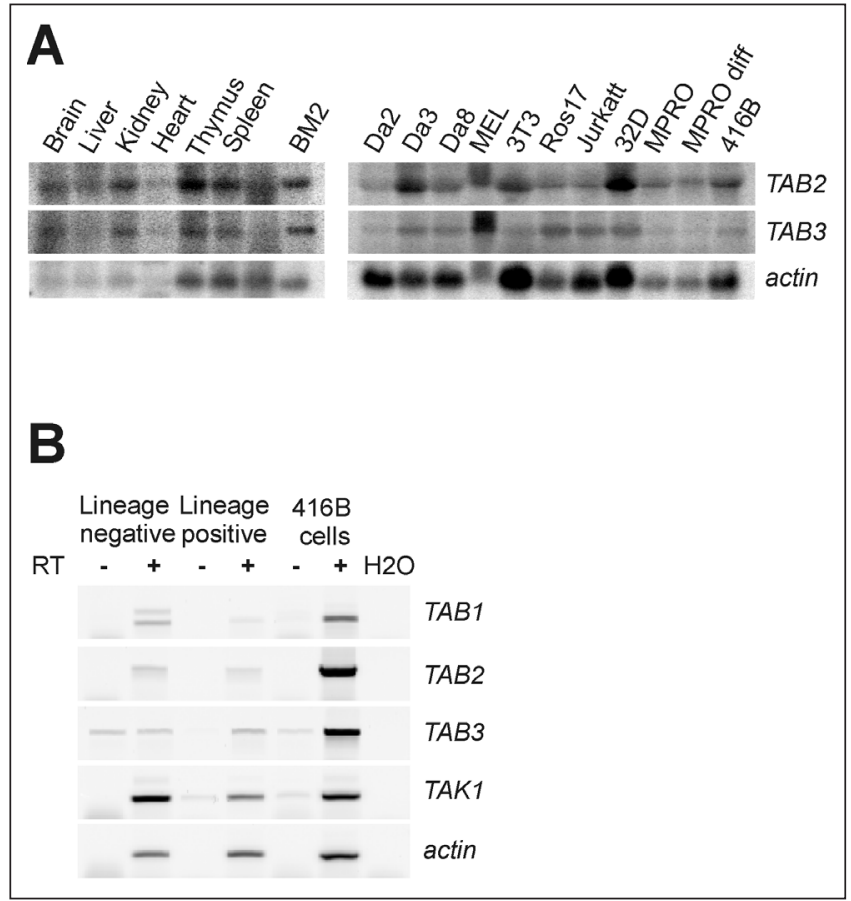

Fig. 2. TAK1 and TAB mRNA expression mouse tissues and adult bone marrow.

A: Northern blot analysis for TAB2 and TAB3 transcripts in cells from several adult mouse tissues and cell lines. B: RT-PCR analysis for TAB1, TAB2, TAB 3 and TAK1 shows that these genes are expressed in both the lineage marker positive (mature hematopoietic cell population) and the lineage marker negative (hematopoietic progenitor cell population) of the adult mouse bone marrow. The 416B mouse hematopoietic cell line is a positive control. RT is reverse transcriptase.

staining. No clear difference in subcellular localization was observed between the truncated and the full-length mTAB2 protein.

\section{mTAB2 protein is expressed in adult mouse tissues}

Previously we reported that the TAB2 gene (Map3k7ip2) is transcribed in all adult mouse tissues ([24]: see also Fig. 2A). To determine whether the mTAB2 protein is expressed similarly, we performed Western blot analysis for mTAB2 using cell lysates from adult mouse tissues. We used two commercially available antibodies successfully used by others to detect endogenous TAB2 $[18,21]$. Both $\alpha$-TAB2 antibodies yielded similar results on Western blots. We detected a protein band with a molecular weight of approximately $76 \mathrm{kD}$ (Fig. 3). Based on the HA-tagged TAB2 protein size (Fig. $1 \mathrm{~A})$, we conclude that the $76 \mathrm{kD}$ band corresponds to TAB2. High levels of TAB2 protein are present in the non-hematopoietic tissues - liver, kidney and heart (Fig. 3, top panels). A longer exposure of the blot was necessary to obtain bands of similar intensity in the other tested tissues. Relatively lower levels of TAB2 protein are present in hematopoietic tissues - BM, spleen and thymus - and the brain and lungs (Fig. 3, lower panels). Thus, TAB2 protein is expressed in all adult mouse tissues examined. 


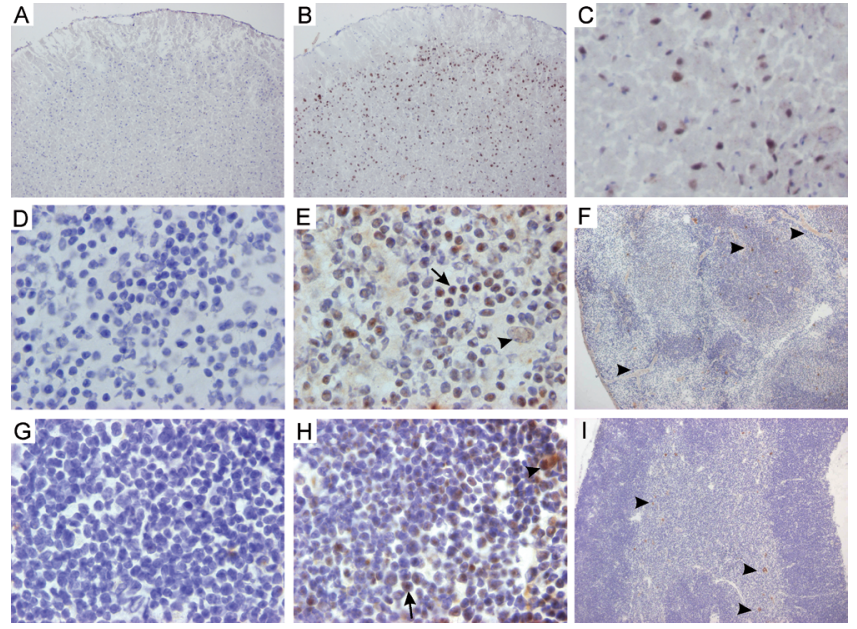

Fig. 4. TAB2 is expressed in the adult mouse in neuronal and hematopoietic tissues. Cryo-sections of adult (A-C) brain, (D-F) spleen and (G-I) thymus. Sections stained with anti-TAB2 (k-20; Santa Cruz) antibody (B, C, E, F, H and I) and negative control sections (no anti-TAB2 antibody; A, D and G) were all counterstained with hematoxylin. Brain TAB2 expression (B) is found in neuronal cells in the outer layers of the cerebellum, except for the outermost layer. (C) TAB2 appears to be localized to the nuclei of the cells. Spleen TAB2 expression is detected in the endothelium of blood vessels (F, arrow heads) and in few scattered cells at the borders of the red and white pulp area and near blood vessels (E; arrow head indicates macrophage-like and arrow indicates lymphoid-like cells). Thymus TAB2 expression ( $\mathrm{H}$ and $\mathrm{I})$ is located to the endothelium of blood vessels and few scattered cells in both the cortex and medulla (arrow heads indicate macrophage/endothelial-like and arrow head indicates lymphoid-like cells). Original $10 \times$ magnification (A, F, I) and 100× magnification (B, C-E, G and H).

\section{Localization of TAB2 in adult mouse tissues}

To more specifically identify and localize the cells expressing TAB2 in adult mouse tissues, we performed immunohistochemistry. Cryosections of several adult mouse tissues were incubated with TAB2 antibody and staining was visualized with the peroxidase substrate DAB chromogen. For these analyses we used the two different TAB2 antibodies which both gave similar TAB2 expression patterns in the tissues tested. Sections were counterstained with hematoxylin to visualize individual cells and the overall morphology of the tissues.

In coronal adult brain sections, TAB 2 is expressed in cells scattered throughout the grey matter of the cerebellum. High expression is observed in cells in the outer layers of the cortex, excluding the outermost layer (Fig. 4B). The expressing cortical cells are mainly neuronal and almost all these cells express TAB2. The highest percentage of TAB2 expressing cells is localized immediately beneath the outermost layer of the cortex. Interestingly, TAB2 staining overlaps with the nuclear hematoxylin staining (Fig. 4C), suggesting that in neuronal cells of the adult brain TAB2 protein is predominantly in the nucleus. In addition, in the midgestation mouse embryo, high TAB2 protein expression is detected in the dorsal root ganglia, the lateral motor column and in nerve tissue near the neural tube (Fig. 5).
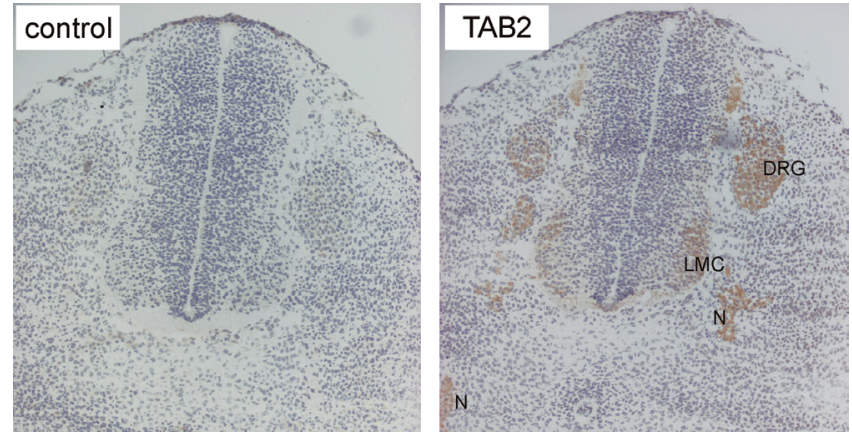

Fig. 5. mTAB2 is expressed in the midgestation neural tube. Immunohistochemistry was performed on transversal cryosections of E11.5 embryos with anti-TAB2 antibody. Prominent TAB2 staining is observed in the dorsal root ganglia (DRG), the lateral motor column (LMC) and in nerve tissue $(\mathrm{N})$ near the neural tube.

The spleen is a lymphoid/myeloid tissue that is comprised of the white pulp containing mainly lymphocytes and the red pulp containing mostly erythrocytes and connective tissue. The white pulp is separated from the red pulp by the marginal zone, where macrophages are localized. In the spleen, we observed TAB2 expression in the endothelium of the blood vessels (Fig. 4F, arrow heads), most likely the central arterioles and the trabecular arteries (those vessels containing blood cells entering the spleen). No expression was found in the sinusoids (where blood cells re-enter circulation). Expression of TAB2 at lower levels was also observed in small mononuclear cells scattered throughout the spleen (Fig. 4E, arrows) and in larger granular macrophage-like cells (Fig. $4 \mathrm{E}$, arrow heads). Most of these TAB2 expressing cells are located near blood vessels or at the border of the white and red pulp areas, the marginal zone.

The thymus is also an important lymphoid tissue and can be morphologically separated into the outer cortex, which contains less differentiated lymphocytes, and the inner medulla area, which contains more mature differentiated lymphocytes. Similar to the spleen, TAB2 expression in the thymus is observed in the endothelium of blood vessels (Fig. 4I, arrow heads). Some cells with the morphology of lymphocytes also appear to express low levels of TAB2 (Fig. 4H, arrow). Additionally, a low percentage of cells scattered throughout both the cortex and the medulla regions show TAB2 expression (Fig. 4I, arrow heads). This small population of TAB2 expressing cells may represent macrophages.

TAB2 expression was also found in the endothelium of blood vessels and some bronchioli in the adult lung (low levels; data not shown). No TAB2 expression was observed throughout the myocardial tissue of the heart, but clear TAB2 expression was observed in the endothelium of blood vessels and in some cells located near blood vessels (data not shown). Due to high levels of background staining in the liver and kidney, we could not determine the TAB2 expression pattern in these tissues. Hence, TAB2 protein is highly expressed in endothelial cells lining blood vessels of most adult tissues examined. TAB2 is also expressed in neuronal cells of the adult brain and in some cells in the lymphoid tissues of the adult, the spleen and thymus. 
TAB2 is localized in the cytoplasm of adult bone marrow and spleen cells

Previously, we reported TAB2 protein expression in the major hematopoietic tissues during early mouse development (AGM region and fetal liver) [24]. In this study we investigated whether TAB2 is expressed in the bone marrow (BM), the main site of hematopoiesis in the adult. We performed RT-PCR analysis for TAB1, TAB2 TAB3 and TAK1 expression on adult BM cells separated into two hematopoietic fractions; mature (lineage maker positive) and immature (lineage maker negative) fractions. Expression of all three TAB genes and the TAK1gene was observed in both BM fractions (Fig. 2).

Next we examined adult BM populations for TAB2 protein expression by performing immunofluorescence analysis on cytospins of sorted hematopoietic $\left(\mathrm{CD} 45^{+}\right)$and non-hematopoietic $\left(\mathrm{CD}^{4} 5^{-}\right)$cells. TAB2 protein is expressed in most $\mathrm{BM}$ cells (Fig. 4E and F). It is expressed in both $\mathrm{CD}^{4} 5^{+}$(Fig. $6 \mathrm{I}$ and $\mathrm{J}$ ) and $\mathrm{CD}^{-}$(Fig. 6M and N) cells, but it appears that the $\mathrm{CD}^{-} 5^{-}$fraction expresses higher levels of TAB2. In most cells the TAB2 staining appears to be surrounding the nuclear propidium iodide stain (Fig. $6 \mathrm{E}$, I and J), indicating that TAB2 is localized in the cytoplasmic compartment. However, it is possible that the higher TAB2 expression in a few $\mathrm{CD} 45^{-}$cells (Fig. 6N, arrow) may reflect an additional nuclear localization of TAB2.

Similarly, adult spleen cells unsorted (Fig. 6G and H) and sorted in $\mathrm{CD}_{4} 5^{+}$(Fig. 6K and L) and $\mathrm{CD} 45^{-}$(Fig. $6 \mathrm{O}$ and $\mathrm{P}$ ) fractions were examined for TAB2 expression. Most splenocytes and cells in both the hematopoietic and non-hematopoietic fractions expressed TAB2. TAB2 expression appeared to be localized to the cytoplasm and in rare cells, in the nucleus (Fig. $6 \mathrm{H}$ and $\mathrm{P}$, arrows). Hence, TAB2 protein is expressed in adult $\mathrm{BM}$ and spleen predominantly in the cytoplasmic compartment of most cells.

\section{Discussion}

\section{TAB2 expression pattern in the adult mouse}

By Western blot analysis, we observed widespread TAB2 expression, consistent with our results showing widespread TAB2 mRNA expression in adult mouse tissues. We have shown here that TAB2 protein is expressed in all adult mouse tissues tested. Interestingly, in the adult mouse IL-1RI and IL-1RII proteins are also expressed widely and at high levels in non-hematopoietic tissues and at low levels in lymphoid tissues. Although TAK1 has been subject of a few expression studies [25, 26], until our study, little was known about TAB protein expression patterns.

By immunostaining, we detected TAB2 expression in most adult tissues - in specific cerebellar locations in the brain; in the endothelial cells of blood vessels in the spleen, thymus and heart; and in hematopoietic cells of the BM, spleen and thymus. Interestingly, the spatial expression pattern of TAB2 in adult mouse tissues shows many similarities with the previously reported IL-1R expression pattern [27, 28 ] as well as with the reported in vivo $\mathrm{NFkB}$ activity pattern [23]. Schmidt-Ullrich et al. analysed a transgenic mouse

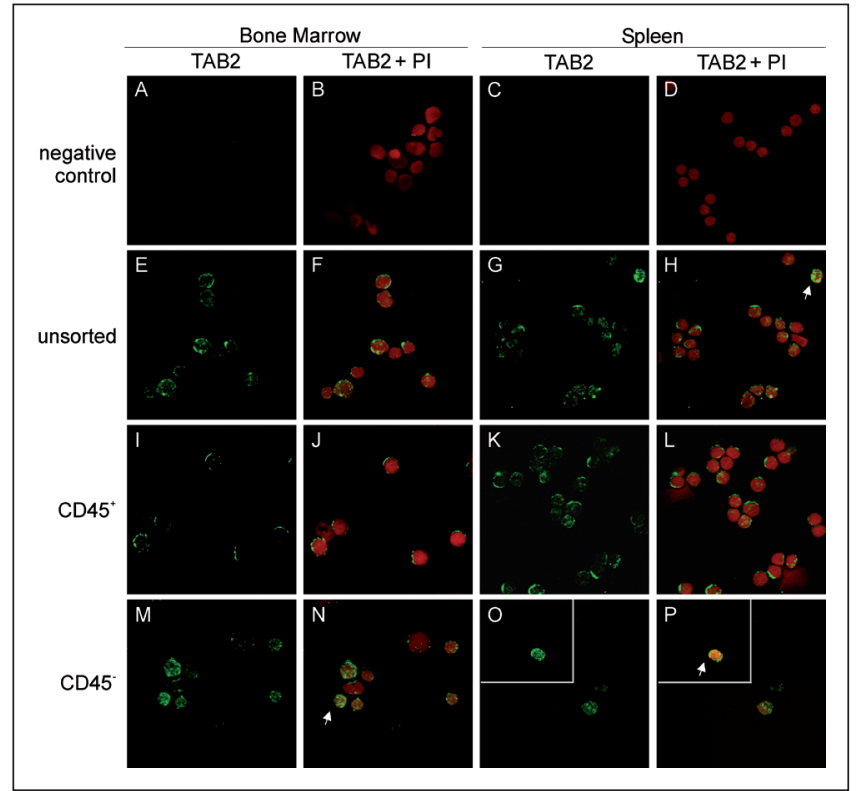

Fig. 6. Intracellular TAB2 localization in adult bone marrow cells. Unsorted $(\mathrm{A}-\mathrm{H})$ and sorted $\mathrm{CD}^{4} 5^{+}(\mathrm{I}-\mathrm{L})$ and $\mathrm{CD} 45^{-}(\mathrm{M}-\mathrm{P})$ adult bone marrow (A, B, E, F, I, J, M, N) and spleen (C, D, G, H, K, L, O, P) cells were prepared as cytospins. Cells were fixed on microscope slides for immunofluorescence staining with anti-TAB2 primary antibody (Affinity BioReagents) and OregonGreen-conjugated secondary antibody (E-P) and Propidium Idodide. OregonGreen fluorescence is shown in all panels and Propidium Iodide fluorescence (red) is shown in panels as indicated. Control unsorted cells (A-D) were stained only with fluorescent secondary antibody and Propidium Iodide. Since very few CD45 cells were obtained from the spleen, two different fields are shown (one field in the inset and one in the background). Most bone marrow and spleen cells are expressing low levels of TAB2 protein. Cells in the CD45 fractions are generally expressing higher levels of TAB2. TAB2 is present in the cytoplasm of most cells, but may be localized in the nucleus of some CD45 cells (arrows in panels H, N, P). Original magnification 400×.

model containing a NFkB-responsive promoter driving expression of the lacZ reporter gene and showed NFkB activity in the adult mouse brain in a scattered pattern in the outer layers of the cerebellum. Moreover, the endothelial cells of several blood vessels and scattered cells in the spleen and thymus were lacZ positive [23]. The expression pattern of NFkB activity also shows striking similarities to the TAB2 expression pattern in the embryo, thus suggesting that TAB2 expression, IL-1 signaling and NFkB activity in vivo may be closely related. [27]. This is supported by our studies in the early mouse embryo showing that the TAB2 expression pattern in the dorsal aorta [24] overlaps with the IL-1RI expression pattern (Orelio et al. submitted).

\section{The subcellular localization of TAB2 suggests multiple functions}

To date, TAB2 was shown to play a role in vitro as an adapter protein in the receptor proximal signalling events of the pro-inflammatory IL-1 and of several TNF-family members. These studies show that TAB2 is involved in the activation of the NFkB pathway via the binding to ubiquitinated 
TRAF2 and TRAF6 in the cytoplasm [14-16, 29-31]. More recent studies with TAB2 deficient cells (Sanjo et al. 2003; Shim et al. 2005) were unable to confirm this crucial role for TAB2 in NFkB activation. This is most likely due to redundancy of TAB2 function with its highly homologous family member, TAB3 $[15,18,31]$. However, TAB2 deficiency in vivo does result in an embryonic lethal phenotype, suggesting that TAB2 plays other important, non-redundant roles [19]. Some insight into the other functions of TAB2 was provided by TAB2 deficient cells. In these cells it has been observed that IL-1 induced NFkB DNA binding activity is higher than in wild type cells, suggesting that TAB2 plays an inhibiting role in the regulation of $\mathrm{NFkB}$ target genes $[19,20]$. Recent studies have confirmed a crucial role for $\mathrm{TAB} 2$ in the repression of NFkB-mediated gene regulation $[21,22]$ and have thereby confirmed that TAB2 has crucial nuclear function.

In this study we have found both a cytoplasmic and the nuclear localization of HA tagged TAB2 in overexpressing $\mathrm{CHO}$ cells. Preliminary studies examining the subcellular localization of endogenous TAB2 protein in cell lines, also show TAB2 protein localization in both compartments (unpublished data). In contrast, our immunostaining data presented here shows mainly a cytoplasmic localization of endogenous TAB2 expression in most adult BM and spleen cells. However, rare cells in the BM (endothelial, myeloid) may also contain nuclear TAB2. Thus, careful analysis of specific lineages of TAB2 expressing cells in specific tissues is required before it can be concluded whether TAB2 expression in one or both of the compartments is related to different functions in vivo. This will also require knowledge of the specific expression pattern of the other TAB family members to determine whether there is functional redundancy.

\section{The TAB2 expression pattern suggests a role in inflammatory-related diseases}

The pro-inflammatory cytokine IL-1 has been shown to play a role in several inflammatory-related diseases, including the neurodegenerative Alzheimer's disease and in vascular diseases such as atherosclerosis [7-9]. More interestingly, based on in vitro studies TAB2 has also been suggested to play a role in the pathogenesis of Alzheimer's disease by regulating NFkB-mediated gene regulation [21]. To date, there are still large gaps in our understanding of the molecular processes that are playing a role in vivo in the pathogenesis of such diseases. Here we show that, similar to the IL-1 receptor, the IL-1 signalling component TAB2 is expressed in the brain and vascular endothelium. Future studies examining the co-expression of TAB2, IL-1 and its receptor and $\mathrm{NFkB}$ activity will determine whether these components together play a pivotal role in the normal physiological regulation as well as in the pathogenesis of these tissues/cell types.

Taken together, the expression pattern and subcellular localization of TAB2 implicates it as an interesting regulator, perhaps serving different in vivo functions in various cell lineages most likely through the IL-1 and NFkB signalling pathways.
Acknowledgements. We thank D. Meijer and M. Jaegle for the HA-tag, anti-HA antibody and HA-Oct6 protein lysate. We thank C. Robin, E. Haak and F. Wahlberg for sorted hematopoietic cell populations, K. van der Horn for technical assistance, D. Meijer, R. Rottier, R. Hendriks and E. Rombouts for assistance in immunostaining analysis. Furthermore we would like to thank all our other colleagues for help and support.

\section{References}

[1] Heimfeld S, Hudak S, Weissman I, Rennick D. The in vitro response of phenotypically defined mouse stem cells and myeloerythroid progenitors to single or multiple growth factors. Proc Natl Acad Sci USA 1991; 88: 9902-6.

[2] Fibbe WE, Goselink HM, Van Eeden G, Van Damme J, Billiau A, Voogt PJ et al. Proliferation of myeloid progenitor cells in human long-term bone marrow cultures is stimulated by interleukin-1 beta. Blood 1988; 72: 1242-7.

[3] Dinarello CA. Biologic basis for interleukin-1 in disease. Blood 1996; 87: 2095-147.

[4] Zucali JR, Moreb J, Gibbons W, Alderman J, Suresh A, Zhang Y et al. Radioprotection of hematopoietic stem cells by interleukin-1. Exp Hematol 1994; 22: 130-5.

[5] Fibbe WE, Hamilton MS, Laterveer LL, Kibbelaar RE, Falkenburg JH, Visser JW et al. Sustained engraftment of mice transplanted with IL-1-primed blood-derived stem cells. J Immunol 1992; 148: 417-21.

[6] O'Neill LA, Dinarello CA. The IL-1 receptor/toll-like receptor superfamily: crucial receptors for inflammation and host defense. Immunol Today 2000; 21: 206-9.

[7] Griffin WS. Inflammation and neurodegenerative diseases. Am J Clin Nutr 2006; 83: 470S-474S.

[8] Merhi-Soussi F, Kwak BR, Magne D, Chadjichristos C, Berti M, Pelli $\mathrm{G}$ et al. Interleukin-1 plays a major role in vascular inflammation and atherosclerosis in male apolipoprotein E-knockout mice. Cardiovasc Res 2005; 66: 583-93.

[9] Krishnaswamy G, Kelley J, Yerra L, Smith JK, Chi DS. Human endothelium as a source of multifunctional cytokines: molecular regulation and possible role in human disease. J Interferon Cytokine Res 1999; 19: 91-104.

[10] Barnes PJ, Karin M. Nuclear factor-kappaB: a pivotal transcription factor in chronic inflammatory diseases. N Engl J Med 1997; 336 : 1066-71.

[11] Denk A, Wirth T, Baumann B. NFkB transcription factors: critical regulators of hematopoiesis and neuronal survival. Cytokine Growth Factor Rev 2000; 11: 303-20.

[12] Shibuya H, Yamaguchi K, Shirakabe K, Tonegawa A, Gotoh Y, Ueno N et al. TAB1: an activator of the TAK1 MAPKKK in TGFbeta signal transduction. Science 1996; 272: 1179-82.

[13] Komatsu Y, Shibuya H, Takeda N, Ninomiya-Tsuji J, Yasui T, Miyado $\mathrm{K}$ et al. Targeted disruption of the Tab1 gene causes embryonic lethality and defects in cardiovascular and lung morphogenesis. Mech Dev 2002; 119: 239-49.

[14] Morlon A, Munnich A, A. S. TAB2, TRAF6 and TAK1 are involved in NF-kappaB activation induced by the TNF-receptor, Edar and its adaptor Edaradd. Hum.Mol.Genetics 2005; 14:3751-3757.

[15] Ishitani T, Takaesu G, Ninomiya-Tsuji J, Shibuya H, Gaynor RB, Matsumoto K. Role of the TAB2-related protein TAB3 in IL-1 and TNF signaling. EMBO J 2003; 22:6277-88.

[16] Takaesu G, Kishida S, Hiyama A, Yamaguchi K, Shibuya H, Irie K et al. TAB2, a novel adaptor protein, mediates activation of TAK1 MAPKKK by linking TAK1 to TRAF6 in the IL-1 signal transduction pathway. Mol Cell 2000; 5: 649-58.

[17] Jin G, Klika A, Callahan M, Faga B, Danzig J, Jiang Z et al. Identification of a human NF-kappaB-activating protein, TAB3. Proc Natl Acad Sci USA 2004; 101: 2028-33.

[18] Cheung PC, Nebreda AR, Cohen P. TAB3, a new binding partner of the protein kinase TAK1. Biochem J 2004; 378: 27-34.

[19] Sanjo H, Takeda K, Tsujimura T, Ninomiya-Tsuji J, Matsumoto K, Akira S. TAB2 is essential for prevention of apoptosis in fetal liver but not for interleukin-1 signaling. Mol Cell Biol 2003; 23: 1231-8. 
[20] Shim JH, Xiao C, Paschal AE, Bailey ST, Rao P, Hayden MS et al. TAK1, but not TAB1 or TAB2, plays an essential role in multiple signaling pathways in vivo. Genes Dev 2005; 19: 2668-81.

[21] Baek SH, Ohgi KA, Rose DW, Koo EH, Glass CK, Rosenfeld MG. Exchange of N-CoR Corepressor and Tip60 Coactivator Complexes Links Gene Expression by NF-kappaB and beta-Amyloid Precursor Protein. Cell 2002; 110: 55-67.

[22] Zhu P, Baek SH, Bourk EM, Ohgi KA, Garcia-Bassets I, Sanjo H et al. Macrophage/Cancer cell interactions mediate hormone resistance by a nuclear receptor derepression pathway. Cell 2006; 124 : 615-29.

[23] Schmidt-Ullrich R, Memet S, Lilienbaum A, Feuillard J, Raphael $\mathrm{M}$, Israel A. NF-kappaB activity in transgenic mice: developmental regulation and tissue specificity. Development 1996; 122: 211728.

[24] Orelio C, Dzierzak E. Identification of 2 novel genes developmentally regulated in the mouse aorta-gonad-mesonephros region. Blood 2003; 101: 2246-9.

[25] Jadrich JL, O'Connor MB, Coucouvanis E. Expression of TAK1, a mediator of TGF-beta and BMP signaling, during mouse embryonic development. Gene Expression Patterns 2003; 3: 131-4.
[26] Dempsey CE, Sakurai H, Sugita T, Guesdon F. Alternative splicing and gene structure of the transforming growth factor beta-activated kinase 1. 2000; 1517: 46-52.

[27] Deyerle KL, Sims JE, Dower SK, Bothwell MA. Pattern of IL-1 receptor gene expression suggests role in noninflammatory processes. J Immunol 1992; 149: 1657-65.

[28] Boraschi D, Rambaldi A, Sica A, Ghiara P, Colotta F, Wang JM et al. Endothelial cells express the interleukin-1 receptor type I. Blood 1991; 78: 1262-7.

[29] Mizukami J, Takaesu G, Akatsuka H, Sakurai H, Ninomiya-Tsuji J, Matsumoto K et al. Receptor Activator of NF-kappaB Ligand (RANKL) Activates TAK1 Mitogen-Activated Protein Kinase Kinase Kinase through a Signaling Complex Containing RANK, TAB2, and TRAF6. Mol Cell Biol 2002; 22: 992-1000.

[30] Jiang Z, Ninomiya-Tsuji J, Qian Y, Matsumoto K, Li X. Interleukin-1 (IL-1) receptor-associated kinase-dependent IL-1-induced signaling complexes phosphorylate TAK1 and TAB2 at the plasma membrane and activate TAK1 in the cytosol. Mol Cell Biol 2002; 22: 7158-67.

[31] Kanayama A, Seth RB, Sun L, Ea CK, Hong M, Shaito A et al. TAB2 and TAB3 activate the NF-kappaB pathway through binding to polyubiquitin chains. $2004 ; 15$ : 535-48.

To access this journal online: http://www.birkhauser.ch/IR 